\title{
The Analysis and Comparison of Energy Efficiency Based on Downlink Massive MIMO System
}

\author{
Zhenzhen Gao, Junxuan Wang, and Yi Li
}

\begin{abstract}
In order to improve the cellular energy efficiency, and without sacrificing the quality of service $(Q \circ S)$ at the user, we minimize the total power consumption. In the paper, under the downlink massive MIMO system, a large number of antennas at base station use to serve multi-users within the same cell. The article derives signal dynamic transmitted power, this problem has shown having a hidden convex optimization, we utilize convex optimization to solve the solutions effectively, and propose optimization algorithm that dynamically assigns energy to users, reduces the loss of energy. Under the same conditions and assumptions, we analyze and compare the performance of conventional maximum ratio transmission (MRT) and zero-forcing (ZF) scheme with optimization algorithm. The simulation results show that they are consistent with the theoretical results, and show the performance of optimization algorithm is better than the $\mathrm{ZF}$, ZF better than the MRT under the same conditions.
\end{abstract}

Index Terms-Maximal-ratio-transmission (MRT), zero-forcing (ZF), optimization algorithm, massive MIMO, downlink transmission power.

\section{INTRODUCTION}

With the development of communication systems, the demand for data services are growing. However, the bandwidth of the wireless communication system is always limited by costs of the radio spectrum. Any increase in the data rate, and without increasing the bandwidth, but the power consumption is reduced, so that the system is more efficient in power efficiency and spectrum efficiency and the cost is relatively low [1]. To meet the demand, in the past these years, massive MIMO, that is massive multiple input multiple output, communication system has become a hot issue, which can greatly improve the spectrum efficiency and energy efficiency [2].

Massive MIMO has the great potential, attracting the interests of many researchers, they focus on the energy efficiency and spectral efficiency of the cellular communication system [2], [3], [4]. Linear precoding scheme plays a major role in signal processing of massive MIMO. In [5], in the case of the downlink massive MIMO, the author analyzed the performance of spectral efficiency and energy efficiency and reliability of link using ZF precoding. In [6], the author compared the vector normalization and the matrix of MRT and ZF precoding in the downlink. In [7], in the case of the achievable data rate in a multi-cell downlink, the authors compared the performance of regularized zero-forcing (RZF) and eigen

Manuscript received April 21, 2015; revised December 12, 2015.

The authors are with Xi'an University of Posts and Telecommunications, xi’an, China (e-mail: $2193195749 @ q q . c o m)$. beamforming (BF). In [8], with ZF, MRT and MMSE, that is Minimum Mean Square Error, the authors analyzed the spectral efficiency in the single-cell downlink of massive MIMO system.

Although the above articles provide good results regarding the performance of linear precoding schemes, they did not compare the quality of service (QoS) and transmit power under the same conditions and at the same time in a single cell downlink scenarios. In [8], the authors analyzed the performance of various precoding schemes of spectral efficiency in the case of a single-cell downlink, fixed the same signal value to interference to noise ratio at the precoders is not a good analysis.

This paper compares and analyzes the performance of MRT and ZF precoding and optimization algorithm in terms of the achievable data rate and the total transmit power in the downlink massive MIMO system in a single cell, under the same assumptions. We assume perfect channel acquisition, our goal is to minimize the total transmitted power. We show that the optimization problem with a hidden convex structure, the solution is proved to dynamically allocate the optimal transmitted power to each user.

\section{SYSTEM MODEL}

\section{A. Channel Model}

As shown in Fig. 1, the system is a base station (BS) equipped with $M$ antennas to serve $K$ single-antenna mobile user, using the same time-frequency resources under a single-cell downlink The channel is Gaussian MIMO channel with assume perfect channel state information.

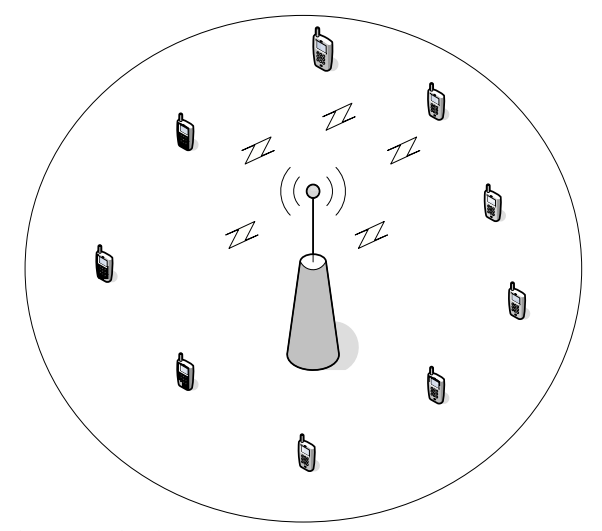

Fig. 1. A single cell downlink massive MIMO system.

The channel model to users $K$ is a block-fading, let $h_{k}$, $h_{k} \in C^{1 \times M}$ denote the channel vector between the BS and the $k_{t h}$ user. The system channel vector between the BS and 
all the users is $H$ as channel matrix. The elements of $H$ are independent and identically distributed complex Gaussian variables with zero mean and unit variance.

The receiver vector is given by:

$$
y=H x+n=H W S+n
$$

where $H$ is a $K \times M$ channel matrix, $x$ is the transmitter vector, $S$ is the receiver signal matrix, and $n$ is noise, and $W$ is a $M \times K$ matrix. The signal received by the $k_{t h}$ user after using the precoding scheme is given by

$$
y_{k}=h_{k} w_{k} s_{k}+\sum_{\substack{i=1 \\ i \neq k}}^{K} h_{k} w_{i} s_{i}+n_{k}
$$

The information symbols are denoted $s_{k}$ from the BS to user $k$ and subject to independent Gaussian distribution with zero mean and unit-variance, that is $s_{k} \sim \mathrm{CN}(0,1)$, for $k=$ $1, \ldots, K$, The precoding vector of $k_{t h}$ user is denoted by $w_{k}$, $w_{k} \in C^{M \times 1}$, and the system formed matrix is $W$.

where $h_{k} w_{k} s_{k}$ is the desired signal, $\sum_{\substack{i=1 \\ i \neq k}}^{K} h_{k} w_{i} s_{i}$ is the interference, that is inter-user interference. The term $n_{k} \sim$ $\mathrm{CN}\left(0, \sigma_{k}^{2}\right)$ is the complex Gaussian noise with zero-mean and variance $\sigma_{k}^{2}$.

The $k_{t h}$ user received signal to interference plus noise ratio can be expressed as [9]

$$
\operatorname{SINR}_{k}=\frac{\left|h_{k} w_{k}\right|^{2}}{\sum_{\substack{i=1 \\ i \neq k}}^{K}\left|h_{k} w_{i}\right|^{2}+\sigma_{k}^{2}}
$$

$S_{N N R_{k}}$ is the $k_{t h}$ user's signal to interference plus noise ratio, which is a function of the precoding vector.

The methods to quantify the communication system performance is the Quality of service (QoS), that is the achievable data rate denoted as $R$, we wish to minimize the total power and to meet the QoS constraints of each user, the QoS constraint is specifying information rate, to make each user achieve data rates, is defined as $\log _{2}\left(1+\operatorname{SINR} R_{k}\right)=\gamma_{k}$, where $\gamma_{k}$ is fixed, achieved by applying successive interference cancellation on the own information symbols and treating the co-user symbol as noise.

In a single-cell downlink massive MIMO system ,each user data rate is up $R$ with perfect channel state information for, that is satisfied with QoS, as follow the $R$ represent data rate $\gamma_{k}$, the achievable data rate can be expressed as

$$
R_{\text {sum }}=K \log _{2}\left(1+\operatorname{SINR}_{k}\right)
$$

\section{B. Maximum-Ratio-Transmission (MRT) Precoding}

MRT is a linear precoding technology, which maximizes the signal gain at the expected user [8]. Assuming $P_{d}$ as the total power of the downlink, the MRT precoding utilized by the $\mathrm{BS}$ is written as

$$
W=H^{H}
$$

For large values of $M$ and $K$, the related $S I N R_{k}$ of MRT precoding is given as [9]

$$
\operatorname{SINR}_{k}^{m r t}=\frac{P_{d} M}{K\left(P_{d}+1\right)}
$$

From (4), the achievable data rate with MRT can be deduced as

$$
R_{\text {sum }}^{m r t}=K \log _{2}\left(1+S I N R_{k}^{m r t}\right)
$$

Substituting (6) into (7), gives

$$
R_{\text {sum }}^{m r t}=K \log _{2}\left(1+\frac{P_{d} M}{K\left(P_{d}+1\right)}\right)
$$

Taking exponential of both sides we have

$$
P_{d}^{m r t}=\frac{K\left(e^{\frac{R_{\text {sum }} \ln 2}{k}}-1\right)}{M-K\left(e^{\frac{R_{\text {sum }} \ln 2}{k}}-1\right)}
$$

\section{Zero-Forcing (ZF) Precoding}

$\mathrm{ZF}$ is a linear precoding technology in which the inter-user interference can be cancelled out at each user [8]. Assuming the total power of the downlink is $P_{d}$, the $\mathrm{ZF}$ precoding employed by the BS is written as

$$
W=H^{H}\left(H H^{H}\right)^{-1}
$$

For large values of $M$ and $K$, the related $S I N R_{k}$ of the $k_{t h}$ user is given as [9]

$$
\operatorname{SINR}_{k}^{z f}=\frac{P_{d}(M-K)}{K}
$$

From (4), the achievable data rate with $\mathrm{ZF}$ can be deduced as

$$
R_{\text {sum }}^{z f}=K \log _{2}\left(1+\operatorname{SINR}_{k}^{z f}\right)
$$

Substituting (11) into (12), gives

$$
R_{\text {sum }}^{z f}=K \log _{2}\left(1+\frac{P_{d}(M-K)}{K}\right)
$$

Taking exponential of both sides we have

$$
P_{d}^{z f}=\frac{K\left(e^{\frac{R_{\text {sum }} \ln 2}{k}}-1\right)}{M-K}
$$




\section{Optimization Algorithm}

From the above equations (6) and (11),the derivation can be seen, the total downlink power is fixed and equally divided to each user, in order to focus the energy transmitting to the user, then proposing dynamic transmit power dividing to each user, we want to minimize the total power and to meet QoS (quality of service) of each user, we propose optimization algorithm, the transmission power consumption can be expressed as [10]:

$$
P=\rho \sum_{k=1}^{K}\left\|w_{k}\right\|^{2}
$$

The aggregation of the emitted powers, $\sum_{k=1}^{K}\left\|w_{k}\right\|^{2}$, each multiplied with a constant $\rho \geq 1$ accounting for the inefficiency of the power amplifier at this transmitter. Meanwhile, considering the actual situation, the power of each antenna is limited, setting by each BS antenna is prone to $L$ power constraints, expressed as follows [11]:

$$
\sum_{k=1}^{K} w_{k}^{H} Q_{l} w_{k} \leq q_{l}, l=1, \ldots \ldots, L
$$

The weighting matrix $Q_{l} \in C^{M \times M}$ is positive semi-definite. The corresponding power limit is $q_{l} \geq 0$, the parameters $Q_{l}, q_{l}$ are fixed, our numerical evaluation considers every antenna constraints of $q$, given by $L=M, q_{l}$ $=q, \forall l$ and $Q_{l}$ with one at $l_{t h}$ diagonal element and zero elsewhere. We are now ready to formulate our optimization problem. We want to minimize the total power consumption while satisfying the QoS constraints and the power constraints, thus

$$
\underset{w_{k}, \forall k}{\min \operatorname{imize}_{i}} \quad P=\rho \sum_{k=1}^{K}\left\|w_{k}\right\|^{2}
$$

subject to $\log _{2}\left(1+S I N R_{k}\right) \geq \gamma_{k}, \forall k$

$$
\sum_{k=1}^{K} w_{k}^{H} Q_{l} w_{k} \leq q_{l}, \forall l
$$

We will prove that (17) can be reformulated as a convex optimization problem and thus is solvable in polynomial time using standard algorithms. Moreover, the power-minimizing solution is self-organizing in the sense that only one or a few transmitters will serve each user. The QoS constraints in (17) are complicated functions of the precoding vectors, making the problem non-convex in its original formulation in [11].

We will prove that it has an underlying convex structure that can be extracted using semi-definite relaxation. To achieve a convex reformulation of (10), we use the notation $W_{k}=w_{k} w_{k}^{H}, \forall k$, This matrix should be a positive semi-definite, denoted as $W_{k} \geq 0$, and have $\operatorname{rank}\left(W_{k}\right) \leq 1$. Note that the rank can be zero, which implies that $W_{k}=0$, we always rewrite (17) compactly as

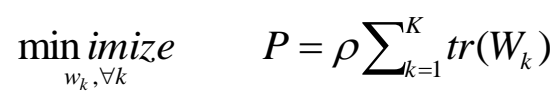

Subject to $\operatorname{rank}\left(W_{k}\right) \leq 1, \forall k$

$$
\begin{gathered}
h_{k}^{H}\left(\left(1+\frac{1}{\tilde{\gamma}_{k}}\right) W_{k}-\sum_{i=1}^{K} W_{i}\right) h_{k} \geq \sigma_{k}^{2}, \forall k \\
\sum_{i=1}^{K} \operatorname{tr}\left(Q_{l} W_{k}\right) \leq q_{l}, \forall l
\end{gathered}
$$

where the QoS targets have been transformed into $\operatorname{SINR}_{k}$ targets of $\tilde{\gamma}_{k}=2^{\gamma_{k}}-1, \forall k$. The non-convex problem transformed into a convex programming, we can use convex optimization to calculate the total transmit power, to minimize the total power, to achieve the highest energy efficiency.

Theorem 1. Consider the semi-definite relaxation of (11) where the rank constraints $\operatorname{rank}\left(W_{k}\right) \leq 1$ are removed. This becomes a convex semi-definite optimization problem. It have an optimal solution $\left\{W_{k}{ }^{*}, \forall k\right\}$ where all matrices satisfy $\operatorname{rank}\left(W_{k}^{*}\right) \leq 1$.

Proof: The proof is given in the appendix.

This theorem shows that the original problem (17) can be solved as a convex optimization problem. This means that the optimal solution is guaranteed in polynomial time [11]; Further structure of the optimal solution can be obtained.

\section{NumERICAL EVALUATIONS}

In order to verify the above theory, we use matlab for theoretical simulation, the number of mobile users is fixed at 10. This section describes the results of the analysis and the algorithm in a scene depicted in the figures shown in Fig. 1, we assume that the small scale Rayleigh fading, $h_{k} \sim C N(0,1)$, correlated matrix is spatially uncorrelated.

We first analyzed the effect of having different numbers of antennas in a single-cell, BS is fixed, 10 users are randomly distributed, where $K=10, \gamma_{k}=2 \mathrm{bit} / \mathrm{s} / \mathrm{Hz}$, the total emitted power increases as the number of antennas becomes smaller, the simulation shown in Fig. 2.

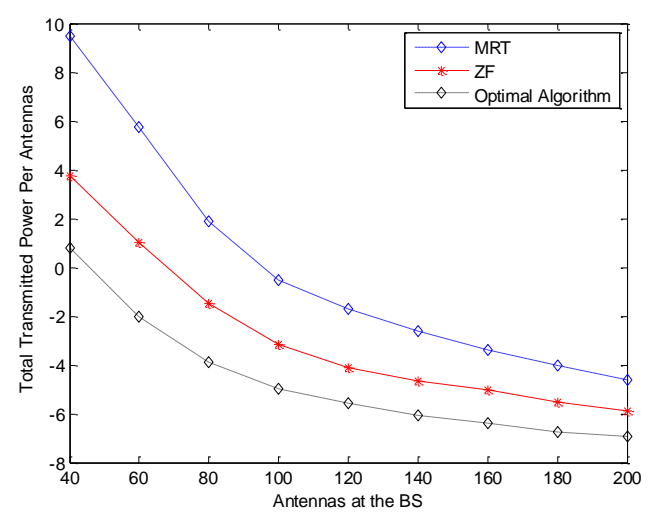

Fig. 2. The total power in the scenario of Fig. 1. We consider different $N_{B S}$, while the QoS constraints are 2 bits $/ \mathrm{s} / \mathrm{Hz}$. 
From Fig. 2, we can see that MRT desired power is maximum, ZF desired power is center, and the optimization algorithm desired power is minimum, the power increases as the number of antennas, the total transmit power along with the change of the data rate varies, where $K=10, M=200$, the simulation as shown in Fig. 3.

From Fig. 3, we can see that MRT desired power is maximum, ZF desired power is center, and the optimization algorithm desired power is minimum, the power increases as the QoS constraints.

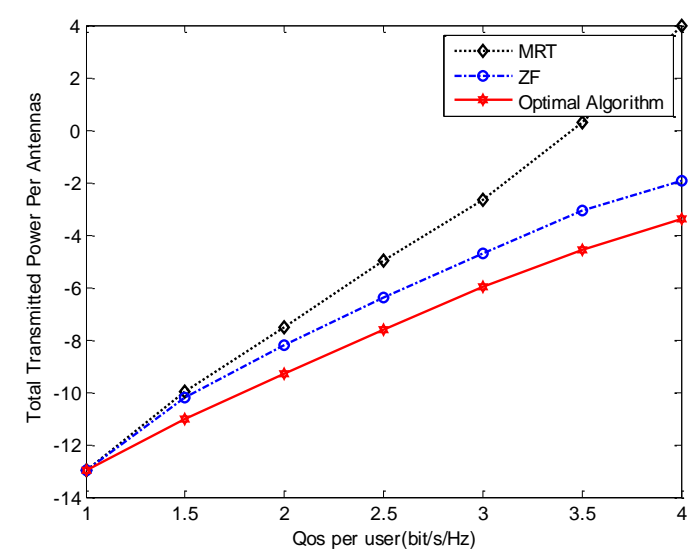

Fig. 3. The total power in the scenario of Fig. 1, with $M=200$, we consider different QoS constraints.

\section{CONCLUSION}

The energy efficiency of the cellular network can be increased at the BS using massive MIMO, we show that the limited power can be minimized by solving a convex optimization problem to achieve, considering the dynamic transmission power, we provide promising results, and the power consumption can be greatly improved.

The results show in Fig. 2 and Fig. 3, with the increasing the number of BS antennas, the required downlink transmission power is reduction. In addition, the performance of the three schemes are compared, then showing that optimization algorithms needed power is less than ZF, ZF needed power is less than the MRT from 40 to 200 BS antenna, which also verified the theoretical results.

\section{APPENDIX}

To prove Theorem 1. The relaxed problem is a semi-definite optimization problem of standard form. Just as shown in [12], there might exist high-rank solutions. But there often exist an optimization solution with $\operatorname{rank}\left(W_{k}^{*}\right) \leq 1, \forall k$. To prove, we suppose there exist an optimal solution $\left\{W_{k}^{* *}, \forall k\right\}$ with $\operatorname{rank}\left(W_{k}^{* * *}\right)>1, \forall k$. We can replace $W_{k}^{* *}$ by any $V \geq 0$, this can always maximize

$$
h_{k}^{H} V h_{k} \quad \text { subject }
$$

$$
\operatorname{tr}(V) \leq \operatorname{tr}\left(W_{k}^{* * *}\right), \operatorname{tr}\left(Q_{l} V\right) \leq \operatorname{tr}\left(Q_{l} W_{k}^{* *}\right), \forall l \text {, not using more }
$$
emitted power than $W_{k}^{* *}$ and $h_{k}^{H} V h_{k} \leq h_{i}^{H} W_{k} h_{i}, \forall i \neq k$ (that is not causing more interference than $W_{k}^{* *}$ ). One solution is $V=W_{k}^{* *}$, but [12] shows problems of that form often have rank-one solutions.

\section{ACKNOWLEDGMENT}

This paper is sponsored by the National Natural Science Foundation of China (GN: 61271276), National High Technology Research and Development Program of China (863 Program) (GN: 2014AA01A703, 2014AA01A705) and "Next-generation broadband wireless mobile communications network" (2014ZX03003005). The author would like to thank anonymous referees for their very constructive comments and corrections of errors.

\section{REFERENCES}

[1] J. Fitzpatrick, "Simulation of a multiple input multiple output wireless system," Dublin City University, April 2004.

[2] E. G. Larsson, F. Tufvesson, O. Edfors, and T. L. Marzetta, "Massive mimo for next generation wireless systems," IEEE Communication Magazine, vol. 52, no. 2, pp.186-195, 2014.

[3] H. Q. Ngo, E. G. Larsson, and T. L. Marzetta, "Energy and spectral efficiency of very large multiuser mimo systems," IEEE Transactions on Communications, vol. 61, no. 4, pp. 1436-1449, 2013.

[4] J. K. Kang, J. Kang, N. Lee, B. M. Lee, and J. Bang, "Minimizing transmit power for cooperative multicell system with massive MIMO," in Proc. the 10th Annual IEEE Cosumer Communications and Networking Conference, 2013, pp. 438-442.

[5] L. Zhao, K. Zheng, H. L. H. Zhao, and W. B. Wang, "Performance analysis for downlink massive MIMO system with ZF precoding," Transactions on Emerging Telecommunications Technologies, vol. 8, no. 3, pp. 390-398, 2014.

[6] Y. G. Lim, C. B. Chae, and G. Caire, "Performance analysis of massive MIMO for cell-boundary users," IEEE Communication Magazine, September 2013.

[7] J. Hoydis, S. T. Brink, and M. Debbah, "Comparison of linear precoding schemes for downlink massive MIMO," in Proc. IEEE ICC - Communications Theory, 2012, pp. 2135-2139.

[8] E. Pakdeejit, "Linear precoding performance of massive MU-MIMO downlink system," Linkopeng University, May 2013.

[9] S. Choi, "Special topics: massive MIMO," presented on Massive MIMO, Yonsei University, June 2012.

[10] S. Cui, A. Goldsmith, and A. Bahai, "Energy-constrained modulation optimization," IEEE Trans. Wireless Commun., vol. 4, no. 5, pp. 2349-2360, 2005.

[11] M. Bengtsson and B. Ottersten, "Optimal and suboptimal transmit beam forming," in Handbook of Antennas in Wireless Communications, L. C. Godara, Ed. CRC Press, 2001.

[12] E. Björnson, N. Jaldén, M. Bengtsson, and B. Ottersten, “Optimality properties, distributed strategies, and measurement-based evaluation of coordinated multicell OFDMA transmission," IEEE Trans. Signal Process., vol. 59, no. 12, pp. 6086-6101, 2011.

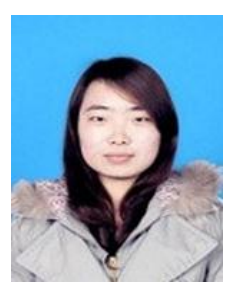

Zhenzhen Gao was born in February 1990, Yulin, Shanxi province, China. Now, she is a graduate student at Xi'an University of Post and Telecommunication, Xi'an, Shannxi, China. Her research direction is wireless broadband communication technology.

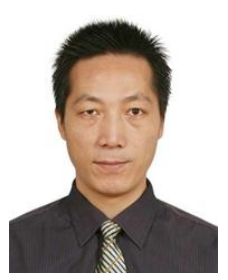

Junxuan Wang was born in 1971. He is a professor as well as a member of Jiu San Society, he has a PhD degree in signal and information processing, he is now working with Xi'an University of Posts and Telecommunications, his research interests are signal and information processing, electronics and communications engineering.

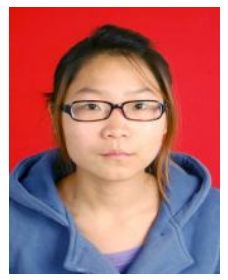

Yi Li was born in December 1992, Weinan, Shanxi province, China. She is now a graduate student in Xi'an University of Post and Telecommunication, Xi'an, Shannxi, China. Her research direction is wireless broadband communication technology. 